\title{
CMB constraints on noncommutative geometry during inflation
}

\author{
Khamphee Karwan ${ }^{a}$ \\ Department of Physics, Faculty of Science, Kasetsart University, Bangkok 10900, Thailand
}

the date of receipt and acceptance should be inserted later

\begin{abstract}
We investigate the primordial power spectrum of the density perturbations based on the assumption that space is noncommutative in the early stage of inflation, and constrain the contribution from noncommutative geometry using CMB data. Due to the noncommutative geometry, the primordial power spectrum can lose rotational invariance. Using the k-inflation model and slow-roll approximation, we show that the deviation from rotational invariance of the primordial power spectrum depends on the size of noncommutative length scale $L_{s}$ but not on sound speed. We constrain the contributions from the noncommutative geometry to the covariance matrix of the harmonic coefficients of the CMB anisotropies using five-year WMAP CMB maps. We find that the upper bound for $L_{s}$ depends on the product of sound speed and slow-roll parameter. Estimating this product using cosmological parameters from the five-year WMAP results, the upper bound for $L_{s}$ is estimated to be less than $10^{-27} \mathrm{~cm}$ at $99.7 \%$ confidence level.
\end{abstract}

PACS. 98.80.Cq Particle-theory and field-theory models of the early Universe - 98.80-k Cosmology

\section{Introduction}

The inflationary cosmology [1,?,?] is the scenario of the very early universe. It provides a successful mechanism for generating nearly scale invariant primordial density perturbations, that give rise to galaxy formation and temperature anisotropies in the $\mathrm{CMB}$ which are in agreement with observation 4. If the period of inflation is sufficiently longer than that required for solving the horizon and flatness problems, such that the wavelengths of perturbations which are observed today emerged from the Planck regime in the early stages of inflation, the physics on trans-Planckian scales should leave an imprint on the primordial density perturbations [5,?]. Here, we consider the imprint of trans-Planckian physics based on noncommutative spacetime.

Near the Planck scale, the properties of spacetime are expected to be modified due to the quantum nature of gravity [7. It has been shown that a consequence of string theory which is a promising candidate of quantum gravity, is that the spacetime is noncommutative [8]

$$
\left[x^{\mu}, x^{\nu}\right]=i \Theta^{\mu \nu}(x)
$$

where $\Theta^{\mu \nu}$ is an antisymmetric tensor.

The influences of spacetime noncommutativity on the feature of power spectrum of primordial fluctuations have been studied by many authors 9] - 16. For the case where $\Theta^{i j}=0$ but $\Theta^{0 i} \neq 0[9, ?, ?]$, the contribution from spacetime noncommutativity can lead to the running of the spectral index of the primordial power spectrum. For

\footnotetext{
a Email:pk_karwan@yahoo.com
}

the case where $\Theta^{i j} \neq 0$ but $\Theta^{0 i}=0$ [12, ?], the primordial power spectrum can become direction-dependent, and consequently the statistics of CMB fluctuations becomes anisotropic. We are interested in this noncommutative geometry induced statistical anisotropy.

Usually, the statistics of the CMB temperature fluctuations is supposed to be isotropic. Hence, if the nonGaussianity of the CMB fluctuations is assumed to be negligible, the statistical properties of the CMB fluctuations will be completely described by the angular power spectrum [17. However, recently there are many attempts to check whether the statistics of the CMB fluctuations is perfectly isotropic by searching for the statistical anisotropy contributions in the CMB sky maps [18,?,?]. In the case where the statistics of the CMB fluctuations is anisotropic, the angular power spectrum does not contain all the information about the statistical properties of the CMB fluctuations even when the Gaussianity of the CMB fluctuations is assumed. Some of the estimators for quantifying the statistical anisotropy contributions in the CMB fluctuations have been proposed in [21,?,?]. According to [18, ?], the statistics of the observed CMB fluctuations does not deviate from isotropy significantly.

In this work, we constrain the contributions from noncommutative geometry to $\mathrm{CMB}$ temperature fluctuations using five-year WMAP CMB maps. In the next section, we compute the power spectrum of primordial density perturbations by taking the spacetime noncommutativity of the form $\Theta^{i j} \neq 0$ and $\Theta^{0 i}=0$. In section 3, we compute the covariance matrix for the harmonic coefficients of the CMB temperature fluctuations $\left\langle a_{l m}^{*} a_{l^{\prime} m^{\prime}}\right\rangle$, and constrain 
the contributions from noncommutative geometry using CMB maps. Finally, we conclude in section 4.

\section{The contributions from noncommutative geometry}

In this section, we investigate the contributions from noncommutative geometry to the primordial power spectrum in the k-inflation model [23,?].

\subsection{The second order action of perturbation}

We start with the general action of the inflaton of the form

$$
S=\frac{1}{2} \int d^{4} x \sqrt{-g}[R+2 P(X, \phi)]
$$

where $\phi$ is the inflaton field and $X=-(1 / 2) \partial_{\mu} \phi \partial^{\mu} \phi$. Here, we have set the reduced Planck mass $(8 \pi G)^{-1 / 2}=1$. To study the evolution of density perturbations during inflation, one expands the action (2) around the homogeneous and isotropic background. In our consideration, we use the ADM metric formalism in which the line element is given by [13,?,?]

$$
d^{2} s=-N^{2} d^{2} t+h_{i j}\left(d x^{i}+N^{i} d t\right)\left(d x^{j}+N^{j} d t\right) .
$$

Using this line element, the action (2) takes the form

$$
\begin{aligned}
S= & \frac{1}{2} \int d^{4} x \sqrt{h} N\left(R^{(3)}+2 P\right) \\
& +\frac{1}{2} \int d^{4} x \sqrt{h} N^{-1}\left(E_{i j} E^{i j}-E^{2}\right),
\end{aligned}
$$

where $h=\operatorname{det}\left(h_{i j}\right), E_{i j}=\frac{1}{2}\left(\dot{h}_{i j}-\nabla_{i} N_{j}-\nabla_{j} N_{i}\right), E=E_{i}^{i}$, a dot denotes the derivative with respect to time and $\nabla_{i}$ is the covariant derivative compatible with $h_{i j}$. The threedimensional Ricci curvature $R^{(3)}$ is computed from the metric $h_{i j}$.

In the ADM formulation $h_{i j}$ and $\phi$ are the dynamical variables, while $N$ and $N^{i}$ are Lagrange multipliers. To compute the perturbed action in the slow-roll approximation, it is convenient to use the uniform curvature gauge in which 25]

$$
\delta \phi \equiv \varphi(t, \mathbf{x}) \quad \text { and } \quad h_{i j}=a^{2} \delta_{i j},
$$

where $a$ is the cosmic scale factor, $\delta \phi(t, \mathbf{x})=\phi(t, \mathbf{x})-\phi_{0}(t)$ is the perturbation in the inflaton field and the subscript 0 represents the background value.

In order to find the second order action for $\varphi$, we first compute the constraint equations for $N$ and $N^{i}$ from the action (44) and solve these equations for $N$ and $N^{i}$ to first order of $\varphi$. the result is 13 .

$$
N=1+\alpha \quad \text { and } \quad N_{i}=\partial_{i} \psi .
$$

Up to the lowest order of slow-roll parameter, the parameters $\alpha$ and $\psi$ can be written as

$$
\alpha=\frac{H \epsilon}{\dot{\phi}_{0}} \varphi \quad \text { and } \quad \partial^{2} \psi=\left(\frac{P_{, \phi_{0}}}{2 H}-\frac{3 H^{2}}{\dot{\phi}_{0}} \epsilon\right) \varphi-\frac{H \epsilon}{\dot{\phi}_{0} c_{s}^{2}} \dot{\varphi},
$$

where $H=\dot{a} / a$ is the Hubble parameter, $\epsilon=-\dot{H} / H^{2}=$ $X P_{, X} / H^{2}$ is the slow-roll parameter, $c_{s}^{2}=P_{, X} / \rho_{, X}$ is the sound speed, the subscript, $X$ denotes a derivative with respect to $X$ and $\rho$ is the energy density of the inflaton.

Substituting equation (6) back in the action and expanding the action to the second order of perturbation, we obtain

$$
\begin{aligned}
\delta S^{(2)}= & \int d^{4} x a^{3}(1+\alpha)\left[P_{, \phi_{0}} \varphi+P_{, \phi_{0} \phi_{0}} \frac{\varphi^{2}}{2}+P_{, X_{0}}\left(\dot{\varphi} \dot{\phi}_{0}\right.\right. \\
& \left.-\left(2 \alpha-3 \alpha^{2}\right) X_{0}-N^{i} \partial_{i} \varphi \dot{\phi}_{0}-\frac{\partial_{\mu} \varphi \partial^{\mu} \varphi}{2}-2 \alpha \dot{\varphi} \dot{\phi}_{0}\right) \\
& \left.+\frac{1}{2} P, X_{0} X_{0}\left(4 \alpha^{2} X_{0}^{2}-4 \alpha X_{0} \dot{\varphi}_{\dot{\phi}_{0}}+\left(\dot{\varphi} \dot{\phi}_{0}\right)^{2}\right)+\ldots\right] \\
& +\frac{1}{2} \int d^{4} x a^{3}(1+\alpha)^{-1}\left(-6 H^{2}\right)+\ldots
\end{aligned}
$$

Keeping the lowest order of slow-roll parameter and second order of $\varphi$, the above action becomes [13]

$$
\begin{aligned}
\delta S^{(2)} & =\int d^{4} x \frac{a^{3}}{2}\left(P_{, X_{0} X_{0}}\left(\dot{\phi}_{0} \dot{\varphi}\right)^{2}-P_{, X_{0}} \partial_{\mu} \varphi \partial^{\mu} \varphi\right), \\
& =\int d^{4} x \frac{a^{3}}{2}\left(\frac{P_{, X_{0}}}{c_{s}^{2}} \dot{\varphi}^{2}-P_{, X_{0}}(\partial \varphi)^{2}\right) .
\end{aligned}
$$

Here, we have used $\rho_{, X}=P_{, X}+2 P_{, X X} X$. From the above calculation, we see that in the slow-roll approximation the second order action of the field perturbation in uniform curvature gauge can be obtained by just expanding Lagrangian $P(X, \phi)$ around the homogeneous field $\phi_{0}$. This is because the terms that are multiplied by the metric perturbation in the perturbed action are subleading in slow-roll parameter [13, ?].

\section{2 noncommutative geometry}

We now study how the noncommutative geometry influences the action of the field perturbations. In order to take the effect of noncommutative geometry into account, we replace the ordinary products in the action with the star products. In curved spacetime, the star product can be expanded as 12 .

$$
\begin{aligned}
f \star g \equiv & \sum_{k=0}^{\infty} \frac{1}{k !}\left(\frac{i}{2}\right)^{k} \Theta^{\mu_{1} \nu_{1}} \cdots \Theta^{\mu_{k} \nu_{k}} \times \\
& \left(D_{\mu_{1}} \cdots D_{\mu_{k}} f\right)\left(D_{\nu_{1}} \cdots D_{\nu_{k}} g\right),
\end{aligned}
$$

where $D_{\mu}$ is the covariant derivative. In this work, we consider the case where $\Theta^{0 i}=0$ while $\Theta^{i j} \neq 0$. The case where $\Theta^{i j} \neq 0$ can arise in the effective theory of D-branes. In some model of D-branes, e.g., a Dp-brane 
on p-dimensional Torus with unequal radii $R_{i}$ 27, the non-commutative parameter $\Theta$ is not necessary equal in all direction. This implies that a preferred direction can occur in space-space noncommutativity. We would like to investigate some effects of this form of isotropy breaking by following the literature 12,13, to choose, Without losing generality, a special frame in which the nonzero components of $\Theta^{\mu \nu}$ are $\Theta^{12}=-\Theta^{21}=L_{s}^{2} / a^{2}$, where $L_{s}$ is the noncommutative length scale [12].

According to the previous section, the terms in the second order action that are multiplied by metric perturbation are subleading in slow-roll parameter. Hence, the noncommutative effect can be incorporated by replacing the products between fields in the Lagrangian by the star products, and expanding the Lagrangian to the second order in the field perturbation. In the calculation of star product, we also ignore the metric perturbation because it gives rise to the terms that are subleading in slow-roll parameter. Since it is possible to study the effect of noncommutative geometry perturbatively [12, we do the calculation up to the lowest non trivial order in $\Theta^{\mu \nu}$. Up to the second order in $\Theta^{\mu \nu}$, the star products of the multiple functions can be written as 13

$$
\begin{aligned}
f_{1} \star \cdots \star f_{n}= & \left(1+\frac{i}{2} \Theta^{\mu \nu} \sum_{a<b} D_{\mu}^{a} D_{\nu}^{b}\right. \\
& \left.-\frac{1}{8} \Theta^{\mu \nu} \Theta^{\rho \sigma} \sum_{a<b, c<d} D_{\mu}^{a} D_{\nu}^{b} D_{\rho}^{c} D_{\sigma}^{d}\right) f_{1} \cdots f_{n},
\end{aligned}
$$

where $a, b, c, d$ run over $1, \ldots, n$.

For illustration, we suppose that $P(X, \phi)$ can be written as $P(X, \phi)=F(\phi) G(X)=\phi^{n} X^{m}$, and use eq. (12) to compute the star product between field varibles. We first consider the case where the derivatives in eq. (12) act on the terms in $G(X)$ only, such that $\delta_{\Theta} P(X, \phi)=$ $F(\phi) \delta_{\Theta} G(x)$, where $\delta_{\Theta}$ denotes the contribution from noncommutative geometry. In this case, the contribution from noncommutative geometry starts to appear at the second order of $\Theta^{\mu \nu}$, i.e.,

$$
\begin{aligned}
\delta_{\Theta} G= & -\frac{1}{8} \Theta^{\mu \nu} \Theta^{\rho \sigma} \sum_{a<b, c<d} D_{\mu}^{a} D_{\nu}^{b} D_{\rho}^{c} D_{\sigma}^{d} G \\
= & -\frac{1}{8} \Theta^{\mu \nu} \Theta^{\rho \sigma} \sum_{a<b, c<d} D_{\mu}^{a} D_{\nu}^{b} D_{\rho}^{c} D_{\sigma}^{d}\left(-\frac{1}{2} \partial_{\gamma} \phi \partial^{\gamma} \phi\right)^{m}, \\
= & -\frac{1}{8} \Theta^{\mu \nu} \Theta^{\rho \sigma}\left(\frac{m(m-1)}{2} X_{0}^{m-2} D_{\mu} D_{\rho} X_{1} D_{\nu} D_{\sigma} X_{1}\right. \\
& -\frac{m}{2} X_{0}^{m-1} D_{\mu} D_{\rho} \partial_{\gamma} \varphi D_{\nu} D_{\sigma} \partial^{\gamma} \varphi \\
& \left.-m(m-1) X_{0}^{m-2} X_{1} D_{\mu} D_{\rho} \partial_{\gamma} \varphi D_{\nu} D_{\sigma} \partial^{\gamma} \phi_{0}\right), \\
= & -\frac{1}{8 F\left(\phi_{0}\right)} \Theta^{\mu \nu} \Theta^{\rho \sigma}\left(\frac{1}{2} P_{, X_{0} X_{0}} D_{\mu} D_{\rho} X_{1} D_{\nu} D_{\sigma} X_{1}\right. \\
& -\frac{1}{2} P_{, X_{0}} D_{\mu} D_{\rho} \partial_{\gamma} \varphi D_{\nu} D_{\sigma} \partial^{\gamma} \varphi \\
& \left.-P_{, X_{0} X_{0}} X_{1} D_{\mu} D_{\rho} \partial_{\gamma} \varphi D_{\nu} D_{\sigma} \partial^{\gamma} \phi_{0}\right),
\end{aligned}
$$

where $X_{1}=-\partial_{0} \phi_{0} \partial^{0} \varphi$ is the firse order perturbation in $X$. In this equation, we omit the terms that are proportional to $D_{\mu} D_{\rho} \partial_{\gamma} \phi_{0} D_{\nu} D_{\sigma} \partial^{\gamma} \phi_{0}$, such as $P_{, X_{0} X_{0}} X D_{\mu} D_{\rho} \partial_{\gamma} \phi_{0} D_{\nu} D_{\sigma} \partial$ because these terms vanish after integration by parts. Using similar consideration, one can show that if the derivatives in eq. (12) act on the terms in $F(\phi)$ only, the noncommutative contribution will be proportional to $P_{,_{0} \phi_{0}}$, and if the derivatives act on the terms in both $F(\phi)$ and $G(X)$, the noncommutative contribution will be proportional to $P_{,_{0} X_{0}}$. In our case, both contributions can be neglected because the terms that are proporttional to $P_{,_{\phi_{0}} \phi_{0}}$ and $P_{, \phi_{0} X_{0}}$ are subleading in slow-roll parameter. Hence, the noncommutative geometry modifies the action in leading order of slow-roll parameter as

$$
\delta_{\Theta} S^{(2)}=\delta S^{(2)}+\delta_{\Theta} S_{X}^{(2)},
$$

where $\delta S^{(2)}$ is the action for the perturbed field in eq. (10) and

$$
\delta_{\Theta} S_{X}^{(2)}=\int d^{4} x a^{3} F\left(\phi_{0}\right) \delta_{\Theta} G
$$

The action $\delta_{\Theta} S_{X}^{(2)}$ can be spit into three parts as $\delta_{\Theta} S_{X}^{(2)}=$ $\delta_{\Theta} S_{X 1}+\delta_{\Theta} S_{X 2}+\delta_{\Theta} S_{X 3}$, where the subscripts $1,2,3$ denote the contribution from the first, second and third terms on the RHS of eq. (13). The expression for $\delta_{\Theta} G$ in eq. (13) is valid for a generic $P(X, \phi)$, because one always do Taylor's expansion of $P(X, \phi)$ around the background when applying the star product in the action for perturbation. Moreover, the action (14) can be obtained by just replacing products with star products in the action (9).

We suppose that $D_{\rho} \Theta^{\mu \nu}=0$, and do integration by parts for the action (15) in the similar way as [12. After doing integration by parts and evalulating the covariant derivatives, each part of $\delta_{\Theta} S_{X}^{(2)}$ becomes

$$
\begin{aligned}
\delta_{\Theta} S_{X 1}= & \int d^{4} x \frac{a H^{2} L_{s}^{4}}{8} P_{, X X} X \partial_{p} \partial_{0} \varphi \partial_{p} \partial^{0} \varphi \\
\delta_{\Theta} S_{X 2}= & \int d^{4} x \frac{a H^{2} L_{s}^{4}}{16} P_{X}\left(\partial_{p} \partial_{\mu} \varphi \partial_{p} \partial^{\mu} \varphi\right. \\
& \left.+2 H \partial_{p} \dot{\varphi} \partial_{p} \varphi-H^{2} \partial_{p} \varphi \partial_{p} \varphi\right), \\
\delta_{\Theta} S_{X 3}= & -\int d^{4} x \frac{a H^{2} L_{s}^{4}}{8} P_{, X X} 2 X \partial_{p} \partial_{0} \varphi\left(\partial_{p} \partial^{0} \varphi+H \partial_{p} \varphi\right),
\end{aligned}
$$

where $p=1,2$. In the above three equations and following calculation, we omit the subscript 0 for $P, \rho$ and $X$ because from now on we will treat them as the background quantities. We note that in the calculation of $\delta_{\Theta} S_{X 3}$ we suppose that $\dot{X}<X$. due to the slow-roll approximation. From these results, we get

$$
\begin{aligned}
\delta_{\Theta} S_{X}^{(2)}= & \frac{1}{16} \int d^{4} x a H^{2} L_{s}^{4} \rho_{, X}\left[\beta \partial_{p} \partial_{0} \varphi \partial_{p} \partial^{0} \varphi\right. \\
& \left.+c_{s}^{2} \partial_{p} \partial_{i} \varphi \partial_{p} \partial^{i} \varphi+2 \beta H \partial_{p} \dot{\varphi} \partial_{p} \varphi-c_{s}^{2} H^{2} \partial_{p} \varphi \partial_{p} \varphi\right]
\end{aligned}
$$

where $\beta=2 c_{s}^{2}-1$. In order to find the evolution equation and the corresponding solution from action (14), it 
is convenient to write the action in terms of the variable $v=z \zeta$, where $z=a \sqrt{2 X \rho, X} / H$ [24]. The variable $\zeta$ is the curvature perturbation in comoving gauge which has a direct connection with the generation of large scale structure and CMB fluctuations. This quantity is related to $\varphi$ through $\zeta=H \varphi / \dot{\phi}_{0}$. Expressing the action (14) in terms of $v$ and expanding $v$ in Fourier space as

$$
v(t, \mathbf{x})=\int \frac{d^{3} \mathbf{k}}{(2 \pi)^{3 / 2}}\left(\mathbf{a}_{\mathbf{k}} v_{\mathbf{k}}(t) \mathrm{e}^{i \mathbf{k} \cdot \mathbf{x}}+h . c .\right),
$$

where $\mathbf{a}_{\mathbf{k}}, \mathbf{a}_{\mathbf{k}}^{\dagger}$ satisfy the canonical commutation relations, the action (14), evaluated in the vacuum after normal ordering, becomes

$$
\begin{aligned}
S= & \int d \eta d^{3} \mathbf{k}\left[\left|v_{\mathbf{k}}^{\prime}\right|^{2}+\left(\frac{a^{\prime \prime}}{a}-c_{s}^{2} k^{2}\right)\left|v_{\mathbf{k}}\right|^{2}\right. \\
& -\frac{L_{s}^{4} H^{2} k_{\perp}^{2}}{8 a^{2}}\left(\beta\left|v_{\mathbf{k}}^{\prime}\right|^{2}-c_{s}^{2} k^{2}\left|v_{\mathbf{k}}\right|^{2}\right. \\
& \left.\left.+\left(\beta\left(6 \frac{a^{\prime \prime}}{a}-10(H a)^{2}\right)-\frac{\beta-1}{2}(H a)^{2}\right)\left|v_{\mathbf{k}}\right|^{2}\right)\right]
\end{aligned}
$$

where the prime denotes derivative with respect to the conformal time $\eta=\int d a / a$ and $k_{\perp}^{2}=k_{1}^{2}+k_{2}^{2}$. In the above action, we have neglected the terms $\dot{c}_{s}^{2}$ and $\dot{\rho}, X$ because these terms are subleading in slow-roll parameter. Up to the lowest order of slow-roll parameter, we can write $a^{2}=1 /(H \eta)^{2}+\mathcal{O}(\epsilon) \simeq 1 /(H \eta)^{2}$ and $a^{\prime \prime} / a \simeq 2 / \eta$ Using these approximations and writing $v_{\mathbf{k}}$ in terms of the new variable

$$
y_{\mathbf{k}}(\eta)=\left(1-\beta \kappa^{2} \eta^{2} k_{\perp}^{2}\right)^{1 / 2} v_{\mathbf{k}}(\eta)
$$

where $\kappa^{2}=H^{4} L_{s}^{4} / 8$ represents the contribution from noncommutative geometry, the action (20) up to first order in $\kappa^{2}$ takes the simple form

$$
\begin{aligned}
S= & \int d \eta d^{3} \mathbf{k}\left[\left|y_{\mathbf{k}}^{\prime}\right|^{2}-\left(c_{s}^{2} k^{2}-\frac{2}{\eta^{2}}\right)\left|y_{\mathbf{k}}\right|^{2}\right. \\
& \left.+\kappa^{2} k_{\perp}^{2}\left(\beta-\frac{\beta-1}{2}\right)\left|y_{\mathbf{k}}\right|^{2}\right] .
\end{aligned}
$$

In the derivation of this action, we suppose that $\kappa^{2} k_{\perp}^{2} \eta^{2}<$ 1 and $\left|c_{s}^{2}\right|$ do not differ much from unity, so that one can expand $1 /\left(1-\beta \kappa^{2} k_{\perp}^{2} \eta^{2}\right) \simeq 1+\beta \kappa^{2} k_{\perp}^{2} \eta^{2}+\ldots$ and neglect the terms that are proportional to $\beta \kappa^{2} k_{\perp}^{2} \eta^{2}$. This implies that this action is valid only if

$$
\eta^{2}<\eta_{i}^{2} \equiv \frac{8}{H^{4} L_{s}^{4} k^{2}}
$$

For a suitable choice of initial conditions, we have $-\infty<$ $\eta<0$ during inflation, so that the perturbation mode $k$ exits the horizon at $\eta_{c}=-1 / k$ and exits the sound horizon at $\eta_{c s}=-1 /\left(k c_{s}\right)$. Hence, we will be able to follow the evolution of the perturbation mode $k$ well before its horizon (sound horizon) exit, i.e., $\eta_{i}<\eta_{c}\left(\eta_{i}<\eta_{c s}\right)$, if the noncommutative length scale is required to be smaller than the Hubble radius, $L_{s}<H^{-1}$.

\subsection{Power spectrum}

In order to study the influence of noncommutative geometry on the behavior of density perturbation, we compute the power spectrum of the curvature perturbation $\zeta$ from action (22). From this action, one can show that the evolution equation for $y_{\mathbf{k}}$ is

$$
y_{\mathbf{k}}^{\prime \prime}+\left(c_{s}^{2} k^{2} \gamma^{2}-\frac{2}{\eta^{2}}\right) y_{\mathbf{k}}=0,
$$

where $\gamma^{2}=1+\kappa^{2} \sin ^{2}(\theta)$ and $\theta=\sin ^{-1}\left(k / k_{\perp}\right)$ denotes the angle between the vectors $\mathbf{k}$ and $\hat{k}_{3}$. Following standard procedure, the solution of eq. (24) is given by [28]

$$
y_{\mathbf{k}}=\frac{e^{-i k c_{s} \gamma \eta}}{\sqrt{2 k c_{s} \gamma}}\left(1+\frac{i}{k c_{s} \gamma \eta}\right)
$$

so that the curvature perturbation is given by

$$
\left|\zeta_{\mathbf{k}}\right|=\left|\frac{v_{\mathbf{k}}}{z}\right| \simeq \frac{\sqrt{2 \pi} H}{m_{p} \sqrt{k^{3} c_{s} \epsilon}}\left(1-\frac{3}{4} \kappa^{2} \sin ^{2}(\theta)\right),
$$

and finally we obtain the primordial power spectrum

$$
\mathcal{P}_{\mathbf{k}}^{\zeta}=\frac{H^{2}}{\pi m_{p}^{2} c_{s} \epsilon}\left(1-\frac{3}{2} \kappa^{2} \sin ^{2}(\theta)\right),
$$

where $m_{p}=G^{-1 / 2}$ is the Planck mass. It can be seen that the obtained power spectrum is direction-dependent due to the noncommutative space. The magnitude of the noncommutative contribution depends on the angle between the vectors $\mathbf{k}$ and $\hat{k}_{3}$, but not on the sound speed. From the expression for $\kappa$, it can be seen that the deviation from rotational invariance of the power spectrum also depends on the noncommutative length scale and Hubble parameter during inflation. One can check that this deviation from rotational invariance is similar to the one that is computed from canonical inflaton field [12. This implies that, in the slow-roll approximation, the effect of noncommutative geometry on primordial power spectrum is the same for both standard inflation and k-inflation model. We note that the slow-roll approximation is required in our calculation because when space is noncommutative, it is not easy to obtain the analytic expression for the primordial power spectrum The amplitude of this power spectrum can be defined as $A_{s}=H^{2} /\left(\pi m_{p}^{2} c_{s} \epsilon\right)$. This quantity as well as $\kappa$ are evaluated when the perturbation mode $k$ exits the horizon. It can be seen that if we neglect the contributions from noncommutative geometry, the above power spectrum gives rise to the usual power spectrum as in 24.

\section{The $\mathrm{CMB}$ constraints}

In this section, we will constrain the contributions from noncommutative geometry to CMB anisotropies using the CMB data. It is well known that if the primordial power 
spectrum is direction-dependent, the statistics of CMB will become anisotropic, i.e., the two-point function of the temperature fluctuations is no longer rotationally invariant. Here, we consider only the two-point function because we assume the non-Gaussianity of the CMB fluctuations to be negligible. In addition to noncommutative geometry, the direction-dependent primordial power spectrum can also be a consequence of many phenomena in the early universe, for example see [29,?,?]. To compare theoretical prediction with the observation, it is convenient to expand the temperature anisotropy into spherical harmonics

$$
\Delta T(\hat{n})=T(\hat{n})-T_{0}=\sum_{l, m} a_{l m} Y_{l m}(\hat{n})
$$

where $T_{0}$ is the mean temperature of the CMB. If we also expand the deviation from rotational invariance into spherical harmonics, the direction-dependent primordial power spectrum can be written as [29, ?]

$$
\mathcal{P}(\mathbf{k})=A(k)\left[1+\sum_{L M} \mathcal{P}_{L M} Y_{L M}(\hat{k})\right] .
$$

Using this primordial power spectrum, the covariance matrix of $a_{l m}$ can be written as

$$
\left\langle a_{l_{1} m_{1}} a_{l_{2} m_{2}}^{*}\right\rangle=\delta_{l_{1} l_{2}} \delta_{m_{1} m_{2}} C_{l_{1}}+\sum_{L M} \Xi_{l_{2} m_{2} L M}^{l_{1} m_{1}} D_{l_{1} l_{2}}^{L M} .
$$

Here, $C_{l_{1}}$ is the CMB angular power spectrum, given by

$$
C_{l_{1}}=(4 \pi)^{2} \int_{0}^{\infty} d k k^{2} A(k)\left[T_{l_{1}}(k)\right]^{2},
$$

where $T_{l_{1}}$ is the CMB transfer function which is taken to be rotationally invariant. It is known that if the two-point function of the temperature anisotropy is not rotationally invariant, the covariance matrix of $a_{l m}$ will not be diagonal. The off diagonal elements of the covariance matrix appear in the second term of eq. (30) This term is given by

$$
D_{l_{1} l_{2}}^{L M}=(4 \pi)^{2}(-i)^{l_{1}-l_{2}} \int_{0}^{\infty} d k k^{2} A(k) \mathcal{P}_{L M} T_{l_{1}}(k) T_{l_{2}}(k),
$$

and

$$
\Xi_{l_{1} m_{1} l_{2} m_{2}}^{l_{3} m_{3}}=\sqrt{\frac{\left(2 l_{1}+1\right)\left(2 l_{2}+1\right)}{4 \pi\left(2 l_{3}+1\right)}} \tilde{\mathcal{C}}_{l_{1} 0 l_{2} 0}^{l_{3} 0} \tilde{\mathcal{C}}_{l_{1} m_{1} l_{2} m_{2}}^{l_{3} m_{3}},
$$

where $\tilde{\mathcal{C}}_{l_{1} m_{1} l_{2} m_{2}}^{l_{3} m_{3}}$ are the Clebsch-Gordan coefficients. Comparing the power spectrum in eq. (29) with the one in eq. (27), we find that the non zero components of $\mathcal{P}_{L M}$ are

$$
\mathcal{P}_{00}=-\frac{4 \sqrt{\pi}}{3} \frac{3}{2} \kappa^{2} \quad \text { and } \quad \mathcal{P}_{20}=\frac{4}{3} \sqrt{\frac{\pi}{5}} \frac{3}{2} \kappa^{2},
$$

We see that the contributions from noncommutative geometry also influence the diagonal elements of the covariance matrix, i.e. they modify the amplitude of $C_{l}$. Substituting $\mathcal{P}_{L M}$ from eq. (34) into eq. (30), we obtain the covariance matrix

$$
\begin{aligned}
\left\langle a_{l_{1} m_{1}} a_{l_{2} m_{2}}^{*}\right\rangle & \simeq C_{l_{1}}\left\{\delta _ { l _ { 1 } l _ { 2 } } \delta _ { m _ { 1 } m _ { 2 } } \left[1+\frac{3 \kappa^{2}}{2} \times\right.\right. \\
& \left.\left(\frac{l_{1}^{2}-m_{1}^{2}}{4 l_{1}^{2}-1}+\frac{\left(l_{1}+1\right)^{2}-m_{1}^{2}}{\left(2 l_{1}+3\right)\left(2 l_{1}+1\right)}-1\right)\right] \\
& -\left(\delta_{l_{1}-2 l_{2}} \delta_{m_{1} m_{2}} \frac{3 \kappa^{2} C_{l_{1} l_{2}}}{2\left(2 l_{1}-1\right)} \times\right. \\
& \left.\left.\sqrt{\frac{\left(\left(l_{1}-1\right)^{2}-m_{1}^{2}\right)\left(l_{1}^{2}-m_{1}^{2}\right)}{\left(2 l_{1}-3\right)\left(2 l_{1}+1\right)}}+l_{1} \leftrightarrow l_{2}\right)\right\}
\end{aligned}
$$

where $C_{l_{1} l_{2}}=(4 \pi)^{2} \int_{0}^{\infty} d k k^{2} A(k) T_{l_{1}}(k) T_{l_{2}}(k) / C_{l_{1}}$.

We next constrain the contributions from noncommutative geometry in this covariance matrix using the fiveyear WMAP foreground-reduced maps 32 . Since the noncommutative geometry also influences the diagonal elements of the covariance matrix and its contribution depends on $H^{4}$, we write the noncommutative contribution in terms of the amplitude of the primordial power spectrum $A_{s}$ as

$$
\frac{3}{2} \kappa^{2}=A_{s}^{2} \Sigma
$$

where

$$
\Sigma=\frac{3}{16} \pi^{2} m_{p}^{4} \epsilon^{2} c_{s}^{2} L_{s}^{4} .
$$

We adopt the procedures in [19] to compute the posterior probability of the parameters $\Sigma$ and $A_{s}$, given the observed temperature anisotropies a,

$$
P(\bar{\sigma} \mid \mathbf{a}) \propto L(\mathbf{a} \mid \bar{\sigma}) P(\bar{\sigma}),
$$

where $\bar{\sigma}=\left\{\Sigma, A_{s}\right\}$ is the set of parameters, $L(\mathbf{a} \mid \bar{\sigma})$ is the likelihood and $P(\bar{\sigma})$ is the prior. Since the galactic contamination cannot be completely removed from some regions of the sky, one does not have the full-sky CMB maps with well-defined error properties. To reduce the galactic contamination, one masks the contaminated regions as

$$
c_{i}=M_{i} \Delta T_{i},
$$

where $i$ is the pixel index, $c_{i}$ is the masked CMB map, $\Delta T_{i}$ is the full-sky map and $M_{i}$ is a mask which is zero at the contaminated points and is one elsewhere. The above relation can be written in harmonic space as

$$
c_{l m}=M_{l m, l^{\prime} m^{\prime}} b_{l^{\prime} m^{\prime}}
$$

where the matrix $M_{l m, l^{\prime} m^{\prime}}$ is given by

$$
M_{l m, l^{\prime} m^{\prime}}=\sum_{L M} M_{L M} \Xi_{L M l^{\prime} m^{\prime}}^{l m}
$$

Here, $c_{l m}, M_{l m}$ and $b_{l m}$ are the spherical harmonic coefficients of $c_{i}, M_{i}$ and $\Delta T_{i}$ respectively. Moreover, due to the instrument noise, the finite beam resolution and the discreteness of the temperature maps, the contributions to the unmasked CMB map come from the sum of the instrument noise with the convolution between the signal 
of the CMB anisotropies and the window function, such that

$$
\Delta \mathcal{T}=W \mathbf{a}+\mathcal{N}
$$

where $W$ is the window function and $\mathcal{N}$ is the instrument noise. We suppose that the contributions from the beam and the pixel asymmetries are negligible. Using eqs. (40) and (42), the covariance matrix of the masked temperature multipoles can be written as

$$
\begin{aligned}
C_{l m, l^{\prime} m^{\prime}}=\sum_{l_{1} m_{1} l_{2} m_{2}} & M_{l m, l_{1} m_{1}}\left[W_{l_{1}}\left\langle a_{l_{1} m_{1}} a_{l_{2} m_{2}}^{*}\right\rangle W_{l_{2}}\right. \\
& \left.+N_{l_{1} m_{1}, l_{2} m_{2}}\right] M_{l_{2} m_{2}, l^{\prime} m^{\prime}}^{*}
\end{aligned}
$$

where $N_{l_{1} m_{1}, l_{2} m_{2}}$ is the pixel noise covariance matrix, given by

$$
N_{l_{1} m_{1}, l_{2} m_{2}}=\Delta a \sum_{L M} \Xi_{L M l_{2} m_{2}}^{l_{1} m_{1}} N_{L M}
$$

Here, $\Delta a$ is the area of each pixel in the temperature map, and $N_{L M}$ is defined as

$$
N_{L M}=\sum_{i} \Delta a \frac{\sigma_{0}^{2}}{n_{i}^{\mathrm{obs}}} Y_{L M}\left(\hat{r}_{i}\right)
$$

where $\sigma_{0}$ is the rms noise of a single observation and $n_{i}^{\text {obs }}$ is the number of observations of pixel $i$.

In order to compute the likelihood, the inversion of the covariance matrix is required. Since the inversion of the large matrix is time consuming, we avoid to inverse the large covariance matrix by writing the likelihood function in terms of the reduced bipolar coefficients instead of $c_{l m}$. The reduced bipolar coefficients are defined as 18

$$
A_{L M}=\sum_{l_{1} m_{1} l_{2} m_{2}}(-1)^{m_{2}} d_{l_{1} m_{1}} d_{l_{2} m_{2}}^{*} \tilde{\mathcal{C}}_{l_{1} m_{1} l_{2}-m_{2}}^{L M},
$$

where $d_{l m}$ are the harmonic coefficients of the temperature anisotropies. For the full-sky and noiseless case, the mean of the reduced bipolar coefficients for the noncommutative k-inflation can be computed using eq. (35), and the non zero components are

$$
\begin{aligned}
\left\langle A_{00}\right\rangle= & \sum_{l}(-1)^{l} C_{l} \sqrt{2 l+1}\left(1-\frac{2}{3} A_{s}^{2} \Sigma\right) \\
\left\langle A_{20}\right\rangle= & -2 A_{s}^{2} \Sigma \sum_{l}(-1)^{l} C_{l} \sqrt{\frac{l(l+1)(2 l+1)}{45(2 l-1)(2 l+3)}} \\
& -2 A_{s}^{2} \Sigma \sum_{l \geq 4}(-1)^{l} C_{l} \sqrt{\frac{2 l(l-1)}{15(2 l-1)}}
\end{aligned}
$$

In the more realistic case, the mask and the instrument noise must be taken into account, so that we compute the reduced bipolar coefficients using eqs. (43) and (35). The result is

$$
\left\langle A_{L M}\right\rangle=\sum_{l_{1} l_{2} m_{1} m_{2}}(-1)^{m_{2}} C_{l_{1} m_{1}, l_{2} m_{2}} \tilde{\mathcal{C}}_{l_{1} m_{1} l_{2}-m_{2}}^{L M} .
$$

It can be seen from the above equation that due to the effect of the mask, $\left\langle A_{00}\right\rangle$ and $\left\langle A_{20}\right\rangle$ will not be the only non zero components of $\left\langle A_{L M}\right\rangle$.

We define

$$
\delta A_{L M}=A_{L M}-\left\langle A_{L M}\right\rangle
$$

and write the covariance matrix for $\delta A_{L M}$ as

$$
\begin{aligned}
& \mathcal{A}_{L M, L^{\prime} M^{\prime}}=\left\langle\delta A_{L M} \delta A_{L^{\prime} M^{\prime}}^{*}\right\rangle \\
= & \sum_{l_{1} m_{1} l_{2} m_{2}} \sum_{l_{3} m_{3} l_{4} m_{4}} \tilde{\mathcal{C}}_{l_{1} m_{1} l_{2} m_{2}}^{L M} \tilde{\mathcal{C}}_{l_{3} m_{3} l_{4} m_{4}}^{L^{\prime} M^{\prime}} \times \\
& \left(C_{l_{1} m_{1}, l_{3} m_{3}} C_{l_{2} m_{2}, l_{4} m_{4}}+C_{l_{1} m_{1}, l_{4} m_{4}} C_{l_{2} m_{2}, l_{3} m_{3}}\right) .
\end{aligned}
$$

We assume for simplicity that posterior probability function for $\delta A_{L M}$ is normally distributed,

$$
\begin{aligned}
P\left(\bar{\sigma} \mid A_{L M}\right) & \propto \frac{\exp \left(-\frac{1}{2} \mathcal{Z}^{2}\right)}{\operatorname{det}^{1 / 2} \mathcal{A}} P\left(A_{s}\right) \\
\mathcal{Z}^{2} & =\sum_{L M L^{\prime} M^{\prime}} \delta A_{L M}\left(\mathcal{A}^{-1}\right)_{L M, L^{\prime} M^{\prime}} \delta A_{L^{\prime} M^{\prime}}^{*}
\end{aligned}
$$

In order to test the above posterior probability function, we repeat some calculation in [19] using this probability function. We have found that the results from this probability function are in agreement with the original results. Here, we have used a flat prior on $\Sigma$ and a Gaussian prior on $A_{s}$. For the Gaussian prior, the mean and variance of $A_{s}$ are taken from the five-year WMAP results 35. Since the contributions from noncommutative geometry mainly appear in the $\left\langle A_{00}\right\rangle$ and $\left\langle A_{20}\right\rangle$, we restrict the multipole index $L$ of $A_{L M}$ to be less than 4 . The data that are used to compute $A_{L M}$ in eq. (50) are the fiveyear WMAP foreground-reduced V2 and W1 differential assembly temperature maps 32 . These maps are masked using the band-limited masks in [19. According to [19, we limit the multipole index of $c_{l m}$ and $M_{L M}$ to be $l \leq 62$ and $L \leq 92$ respectively. The covariance matrix $\mathcal{A}_{L M, L^{\prime} M^{\prime}}$ in eq. (51) is computed using eqs. (43) and (35). Since the noncommutative geometry does not affect the cosmic evolution after the inflationary epoch, we can use CMBEASY 34 to compute the CMB transfer function and $C_{l}$ by supposing that the cosmic evolution after inflation obeys the $\Lambda \mathrm{CDM}$ model whose parameters are taken from the best fit value of the five-year WMAP results 35. However, recall that the amplitude of the primordial power spectrum is treated as a free parameter. We note that the parallel computing of covariance matrices $C_{l_{1} m_{1}, l_{2} m_{2}}$ and $\mathcal{A}_{L M, L^{\prime} M^{\prime}}$ can be easily implemented in the Healpix package [33. We compute the posterior probability function for $\bar{\sigma}$ and marginalize it over $A_{s}$ to obtain the marginalized posterior probability function for $\Sigma$. The marginalized posterior probability functions for $\Sigma$ obtained from $V 2$ and $W 1$ maps have a peak at negative $\Sigma$, which can occur if $c_{s}^{2}$ is negative. However, we cannot use the above analysis to constrain parameters $L_{s}, c_{s}^{2}$ and $\epsilon$ simultaneously due to degeneracy among these parameters. Since we are interested in the constraint on $L_{s}$, we suppose that 


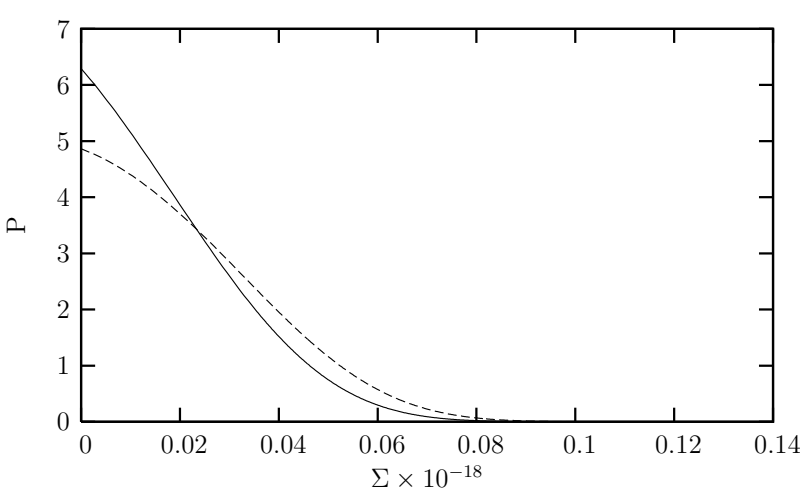

Fig. 1. The marginalized posterior probability functions for $\Sigma$. The solid line represents the probability function from $W 1$ map, while the long dashed line represents the probability function from $V 2$ map. Here, $\Sigma$ is restricted to be positive.

Table 1. The upper bound for the parameter $\Sigma \times 10^{-18}$ from $W 1$ and $V 2$ maps.

\begin{tabular}{c|ccc}
\hline & $68 \% \mathrm{CL}$ & $95 \% \mathrm{CL}$ & $99.9 \% \mathrm{CL}$ \\
\hline $\mathrm{W} 1$ & 0.024 & 0.048 & 0.070 \\
$\mathrm{~V} 2$ & 0.029 & 0.055 & 0.078 \\
\hline
\end{tabular}

values of $c_{s}^{2}$ and $\epsilon$ are known. For canonical scalar field, $\Sigma>0$ because $c_{s}^{2}=1$. Hence, we restrict ourselves to the case where $\Sigma>0$, i.e. $c_{s}^{2}>0$. In this case, the confidence intervals for $\Sigma$ can be obtained from the areas under the curves of The marginalized posterior probability functions in figure 1.

In order to estimate the upper bound for $L_{s}$ from the upper bound for $\Sigma$ in table 1, we further assume that the values of the product $\epsilon c_{s}$ are known precisely, so that the upper bound for $L_{s}$ can be written as $L_{s}<$ $1.4 \times 10^{4}\left[\epsilon^{2} c_{s}^{2} m_{p}^{4}\right]^{-1 / 4} \mathrm{Gev}^{-1} \sim 1.4 \times 10^{-28}\left[\epsilon c_{s}\right]^{-1 / 2} \mathrm{~cm}$ at $99.7 \%$ confidence level. It can be seen that if inflaton evolves more slowly, the upper bound for $L_{s}$ will increase. As shown in 24, the ratio of the tensor to scalar perturbations amplitudes $r$ depends on $\epsilon c_{s}$. We roughly estimate the values of $\epsilon c_{s}$ using the values of $r$ from the five-year WMAP results, and obtain $L_{s}<10^{-27} \mathrm{~cm}$ at $99.7 \%$ confidence level.

\section{Conclusions}

In this work, we study the effect of noncommutative geometry on the rotational invariance of the primordial power spectrum, and constrain the contributions from this effect using CMB data. In the slow-roll approximation, the deviation from rotational invariance of the primordial power spectrum due to the noncommutative effect depends on the factor $3 H^{4} L_{s}^{4} / 16$, where $H$ is evaluated at the time when perturbation mode $k$ crosses the horizon during inflation. Although this result is obtained using k-inflaton, it is similar to the one that is computed from canonical inflaton field [12. This implies that, in the slow-roll approximation, the effect of noncommutative geometry on primordial power spectrum is the same for both standard inflation and k-inflation model.

Since the primordial power spectrum is direction-dependent in our consideration, the covariance matrix for the harmonic coefficients of the CMB temperature anisotropies has off diagonal elements. In our case, these off diagonal elements arise from the noncommutative geometry contributions. As is well known, this implies that statistics of the CMB anisotropies become anisotropic. The noncommutative geometry also contributes to the diagonal elements of the covariant matrix suggestting that the noncommutative contribution also modifies the amplitude of the $\mathrm{CMB}$ angular power spectrum.

Both contributions from noncommutative geometry are simultaneously constrained using five-year WMAP foregroundreduced V2 and W1 maps. The upper bound for the quantity $L_{s}\left[\epsilon c_{s}\right]^{1 / 2}$ is approximately $1.4 \times 10^{-28} \mathrm{~cm}$ at $99.7 \%$ confidence level. If we suppose that the values of $\epsilon c_{s}$ are known precisely, the upper bound for the noncommutative length scale can be written as $L_{s}<1.4 \times 10^{-28}\left[\epsilon c_{s}\right]^{-1 / 2} \mathrm{~cm}$ at $99.7 \%$ confidence level. This shows that if inflaton evolves more slowly, the upper bound for $L_{s}$ will increase. Estimating the values of $\epsilon c_{s}$ using the tensor to scalar perturbations amplitudes from the five-year WMAP results, we obtain $L_{s}<10^{-27} \mathrm{~cm}$ at $99.7 \%$ confidence level.

\section{Acknowledgments}

The author would like to thank T. Souradeep for instructive conversations, and A. Ungkitchanukit for comments on the manuscript. He would also like to thank P. Wongjun for his checking of some parts of the calculations, P. Burikham and A. Chatrabhuti for their comments and suggestions. The numerical calculation was performed using the computer cluster of Department of Physics, Kasetsart University He acknowledges the use of the Legacy Archive for Microwave Background Data Analysis (LAMBDA) and HEALPix package. This work is supported by Thailand Research Fund (TRF).

\section{References}

1. A. H. Guth, Phys. Rev. D23, 347 (1981).

2. A. D. Linde, Phys. Lett. B108, 389 (1982). 
3. A. Albrecht and P. J. Steinhardt, Phys. Rev. Lett. 48, 1220 (1982).

4. E. Komatsu et al, Astrophys. J. Suppl. 180, 330 (2009), arXiv:0803.0547.

5. R. H. Brandenberger and J. Martin, Mod. Phys. Lett. A16, 999 (2001), arXiv:astro-ph/0005432.

6. J. Martin and R. H. Brandenberger, Phys. Rev. D63, 123501 (2001), arXiv:hep-th/0005209 ].

7. R. J. Szabo, Phys. Rept. 378, 207 (2003), arXiv:hep-th/0109162.

8. N. Seiberg and E. Witten, JHEP 9909, 032 (1999), arXiv:hep-th/9908142.

9. R. H. Brandenberger and Pei-Ming Ho, Phys. Rev. D66, 023517 (2002), arXiv:hep-th/0203119.

10. S. Tsujikawa, R. Maartens and R. H. Brandenberger, Phys. Lett. B574, 141 (2003), arXiv:astro-ph/0308169.

11. Qing-Guo Huang and M. Li, JHEP 0306, 014 (2003), arXiv:hep-th/0304203.

12. F. Lizzi, G. Mangano, G. Miele and M. Peloso, JHEP 0206, 049 (2002), arXiv:hep-th/0203099.

13. K. Fang, B. Chen and W. Xue, Phys. Rev. D77, 063523 (2008), arXiv:0707.1970.

14. E. Akofor, A. P. Balachandran, S. G. Jo, A. Joseph and B. A. Qureshi, JHEP 0805, 092 (2008), arXiv:0710.5897.

15. Yi-fu Cai and Yun-Song Piao, Phys. Lett. B657, 1 (2007), arXiv:gr-qc/0701114.

16. E. Akofor, A. P. Balachandran, A. Joseph, L. Pekowsky and B. A. Qureshi, Phys. Rev. D79, 063004 (2009), arXiv:0806.2458.

17. C. Armendariz-Picon, JCAP 0603, 002 (2006), arXiv: astro-ph/0509893.

18. A. Hajian and T. Souradeep, Phys. Rev. D74, 123521 (2006), arXiv:astro-ph/0607153.

19. C. Armendariz-Picon and L. Pekowsky, Phys. Rev. Lett. 102, 031301 (2009), arXiv:0807.2687.

20. N. E. Groeneboom and H. K. Eriksen, Astrophys. J. 690, 1807 (2009), arXiv:0807.2242.

21. A. Hajian and T. Souradeep, Astrophys. J. 597, L5 (2003), arXiv:astro-ph/0308001.

22. A. R. Pullen and M. Kamionkowski, Phys. Rev. D76, 103529 (2007), arXiv:0709.1144.

23. C. Armendariz-Picon, T. Damour and V. F. Mukhanov, Phys. Lett. B458, 209 (1999), arXiv:hep-th/9904075.

24. J. Garriga and V. F. Mukhanov, Phys. Lett. B458, 219 (1999), arXiv:hep-th/9904176.

25. J. Maldacena, JHEP 0305, 013 (2003), arXiv: astro-ph/0210603

26. X. Chen, Min-Xin Huang, S. Kachru and G. Shiu, JCAP 0701, 002 (2007), arXiv:hep-th/0605045.

27. Chong-Sun Chu and Pei-Ming Ho, Nucl. Phys. B550, 151 (1999), arXiv:hep-th/9812219.

28. A. Riotto, Trieste 2002, Astroparticle physics and cosmology, 317 (2002), arXiv:hep-ph/0210162.

29. L. Ackerman, S. M. Carroll and M. B. Wise, Phys. Rev. D75, 083502 (2007), arXiv:astro-ph/0701357.

30. C. G. Boehmer and D. F. Mota, Phys. Lett. B663, 168 (2008), arXiv:0710.2003.

31. T. S. Koivisto and D. F. Mota, JCAP 0808, 021 (2008), arXiv:0805.4229.

32. G. Hinshaw et al, Astrophys. J. Suppl. 180, 225 (2009), arXiv:0803.0732

33. K. M. Gorski, E. Hivon, A. J. Banday, B. D. Wandelt, F. K. Hansen, M. Reinecke and M. Bartelman, Astrophys. J. 622, 759 (2005), arXiv:astro-ph/0409513.
34. M. Doran, JCAP 0510, 011 (2005), arXiv:astro-ph/0302138.

35. J. Dunkley et al, Astrophys. J. Suppl. 180, 306 (2009), [arXiv:0803.058] 